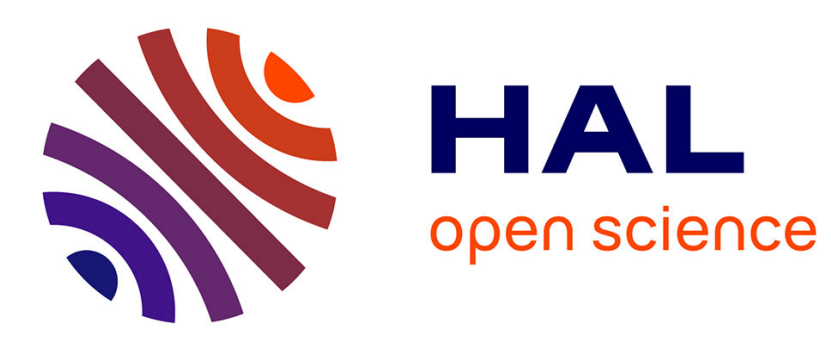

\title{
Système des démonstratifs médiévaux et exemples de stratégies communicatives
}

Céline Guillot

\section{To cite this version:}

Céline Guillot. Système des démonstratifs médiévaux et exemples de stratégies communicatives. Journal of French Language Studies, 2012, 23 (2), pp.221-242. halshs-00854340

HAL Id: halshs-00854340

https://shs.hal.science/halshs-00854340

Submitted on 22 Oct 2014

HAL is a multi-disciplinary open access archive for the deposit and dissemination of scientific research documents, whether they are published or not. The documents may come from teaching and research institutions in France or abroad, or from public or private research centers.
L'archive ouverte pluridisciplinaire HAL, est destinée au dépôt et à la diffusion de documents scientifiques de niveau recherche, publiés ou non, émanant des établissements d'enseignement et de recherche français ou étrangers, des laboratoires publics ou privés. 


\section{Journal of French Language Studies}

http://journals.cambridge.org/JFL

Additional services for Journal of French

\section{Language Studies:}

Email alerts: $\underline{\text { Click here }}$

Subscriptions: $\underline{\text { Click here }}$

Commercial reprints: $\underline{\text { Click here }}$

Terms of use : $\underline{\text { Click here }}$

\section{Système des démonstratifs médiévaux et exemples de stratégies communicatives}

CÉLINE GUILLOT

Journal of French Language Studies / Volume 23 / Issue 02 / July 2013, pp 221 - 242

DOI: 10.1017/S0959269512000245, Published online: 08 August 2012

Link to this article: http://journals.cambridge.org/abstract S0959269512000245

How to cite this article:

CÉLINE GUILLOT (2013). Système des démonstratifs médiévaux et exemples de stratégies communicatives. Journal of French Language Studies, 23, pp 221-242 doi:10.1017/S0959269512000245

Request Permissions : $\underline{\text { Click here }}$ 


\title{
Système des démonstratifs médiévaux et exemples de stratégies communicatives
}

\author{
CÉLINE GUILLOT
}

ENS de Lyon-UMR 5191 ICAR, Institut universitaire de France

(Received October 20II; revised June 20I2; first published online 8 August 20I2)

\section{RÉSUMÉ}

Notre recherche porte sur la valeur sémantique des deux séries de démonstratifs médiévaux, CIST et CIL, aux $\mathrm{I}_{2}{ }^{\mathrm{e}}$ et $\mathrm{I}_{3}{ }^{\mathrm{e}}$ siècles. En nous fondant sur la théorie de la sphère personnelle du locuteur, nous montrons que l'usage des deux paradigmes médiévaux repose sur les stratégies communicatives du locuteur, qui se positionne par rapport à son allocutaire en choisissant d'utiliser telle ou telle forme. Pour étayer notre hypothèse, nous étudions plus spécifiquement les SN démonstratifs prédicatifs en français médiéval. Nous montrons également que notre approche permet de mieux comprendre certains effets du démonstratif du français moderne.

\section{INTRODUCTION}

Notre étude s'inscrit dans le cadre des recherches qui sont menées actuellement sur la valeur sémantique des démonstratifs en ancien français et son évolution jusqu'en français moderne. Dans cette perspective, on s'intéressera ici plus spécifiquement au français écrit entre le $\mathrm{II}^{\mathrm{e}}$ et le $\mathrm{I}^{\mathrm{e}}{ }^{\mathrm{e}}$ siècle, en établissant quelques parallèles avec le français moderne.

Le système médiéval est d'abord organisé autour de deux paradigmes (CIST et CIL $^{1}$ ), hérités de deux des quatre séries latines ${ }^{2}$ (ISTE pour CIST et ILLE pour CIL), puis il s'enrichit d'un troisième paradigme (CE) entre la fin du $\mathrm{I}^{\mathrm{e}}$ et le $\mathrm{I}_{3}{ }^{\mathrm{e}}$ siècle. On suppose que cette dernière série, qui ne provient pas du latin, neutralise dès le départ la valeur sémantique qui permet d'opposer CIST à CIL. On sait par ailleurs que l'opposition sémantique entre CIST et CIL va progressivement s'affaiblir, alors que, dans le même temps, le système se réorganisera autour d'une nouvelle opposition non plus sémantique mais morphosyntaxique. La fin de l'évolution aboutit donc entre le $\mathrm{I} \sigma^{\mathrm{e}}$ et le $\mathrm{I} 7^{\mathrm{e}}$ siècle à deux paradigmes spécialisés, l'un comme pronom, l'autre comme déterminant, à l'intérieur d'un système relativement pauvre du point de vue sémantique (seuls les suffixes -ci

${ }^{1}$ On utilise généralement les majuscules pour désigner un paradigme de formes. On suivra cet usage dans la suite de l'article.

2 On inclut généralement l'anaphorique is, ea, id, dans la série des démonstratifs latins. 
et -là, souvent absents avec le déterminant, continuent de distinguer deux séries de formes en français moderne).

Dans cette étude, on s'appuie sur l'hypothèse que, entre le $\mathrm{I} \mathrm{I}^{\mathrm{e}}$ et le $\mathrm{I} 3^{\mathrm{e}}$ siècle, le système des démonstratifs est orienté par rapport à la personne du locuteur, comme le suppose la thèse de la sphère personnelle du locuteur développée par C. Marchello-Nizia. La question qui est au centre de notre article est celle du couple locuteur/allocutaire et des relations qu'on peut établir entre le système des démonstratifs médiévaux et les deux personnes de l'interlocution. On essaiera de montrer en quoi le choix d'une série de formes repose sur la relation interpersonnelle des participants de l'interaction et/ou en quoi ce choix peut être motivé par la stratégie communicative du locuteur. En d'autres termes, on verra en quoi le choix d'une forme nous renseigne sur la façon dont le locuteur se positionne par rapport à son allocutaire tantôt en se l'associant, tantôt en s'en dissociant.

\section{THÉORIE De LA SPHÈre PERSONNELle DU LOCUTEUR}

\subsection{Présentation de la théorie}

Fondée sur la notion de sphère personnelle du locuteur, la théorie élaborée par Marchello-Nizia (2003, 2004, 2005, 2006a et b) assigne au locuteur une place centrale dans le système des démonstratifs médiévaux. Elle considère en effet que la valeur sémantique des deux séries CIST et CIL est subjective et basée sur la seule appréciation du locuteur. Celui-ci a toujours le choix d'utiliser l'une ou l'autre série en fonction de la visée communicative de son énoncé: en utilisant l'une des formes de CIST, il indique au destinataire qu'il inclut le référent dans sa sphère personnelle, en choisissant l'une des formes de CIL il indique au contraire qu'il l'exclut ou le rejette hors de sa sphère.

La sphère du locuteur n'est aucunement liée à la position spatiale du référent par rapport au locuteur. Il s'agit plutôt d'une sorte de zone très abstraite, qui comporte tout ce que le locuteur présente comme le concernant ou étant directement relié à lui et qu'il construit de manière dynamique dans son propre discours. Elle comprend généralement les objets qui lui appartiennent, les parties de son corps, les membres de sa famille, ses proches, ceux qu'il aime et chérit. On opposera ainsi les énoncés (I) et (2) :

(I) « Biax amis, fet Perceval, je te pri [...] que tu cel cheval me prestes tant que je aie ateint un chevalier qui ci s'en vet. " (Queste del saint Graal p. 88) "Cher ami, dit Perceval, je te demande de me prêter ce(/ton) cheval le temps que je rattrape un chevalier qui vient de s'enfuir."

(2) «Deus, dist Guillelmes, com vos dei graciier

De cest cheval que j'ai ci guaaignié ! " (Couronnement de Louis, v. I I47)

"Dieu, dit Guillaume, combien je vous dois de gratitude pour ce cheval que j'ai gagné !"

Dans le premier cas, le locuteur demande à son interlocuteur de lui prêter son cheval, dans le second, le cheval est bien présenté par le locuteur comme lui 
appartenant. La sphère inclut donc de façon large tout ce avec quoi le locuteur revendique un lien personnel fort et ce sur quoi il a une certaine emprise.

\subsection{Sphère du locuteur et sphère de l'allocutaire}

Les recherches en corpus de C. Marchello-Nizia lui ont permis d'interpréter un grand nombre d'énoncés qui comportent simultanément des formes de CIST et de CIL, et dans lesquels l'opposition entre les deux séries de formes permet d'opérer une distinction entre deux personnes dont l'une est aimée du locuteur et l'autre non. Dans le roman d'Eneas par exemple, Didon amoureuse d'Enée ne peut cependant trahir la fidélité qu'elle a jurée à Sychée, son époux défunt. Dans son discours à sa soeur Anna, elle oppose les deux hommes en utilisant le pronom celui pour désigner son ancien mari, et le pronom cestui pour Enée, objet présent de son amour:

(3) «Se por ce non qu'a mon espos

pramis m'amor a mon vivant,

De lui fë̈sse mon amant ; mais quant je l'ai celui donee, ja par cestui n' iert violee. " (Roman d'Eneas, v. I307-I308)

"N'eût été le fait que j'ai promis mon amour à mon époux pour toute ma vie, j'aurais fait de lui mon amant; mais dès lors que je l'ai [mon amour] donné à celui-là, jamais cet amour ne sera violé par celui-ci. "

Dans l'exemple suivant, le référent qui est rejeté hors de la sphère du locuteur se confond avec le destinataire du message:

(4) «Se voz de ceste ne voz poéz oster,

Je voz ferai celle teste coper ». (Ami et Amile, v. 752-753)

"Si vous ne pouvez vous disculper à propos de celle-ci, je vous ferai couper la tête. "

Le père de Bélissant s'adresse ici à Amile qui a passé la nuit avec sa fille. Le roi choisit le pronom ceste pour désigner sa fille chérie, mais en menaçant l'amant qui l'a déshonorée il utilise une forme de la série CIL. Celle teste indique bien qu'il s'agit de la tête du destinataire, et en même temps, par le choix de cette expression et le contraste opéré avec ceste, le roi marque clairement son opposition au jeune-homme et son animosité envers lui.

Une situation de communication particulièrement intéressante est celle où la façon dont le locuteur présente le référent est moins conditionnée par sa relation avec le référent que par sa relation avec l'allocutaire. Tel est le cas par exemple de l'énoncé suivant:

(5) Li empereres respunt par maltalant:

"Alez sedeir desur cel palie blanc!

N'en parlez mais, se jo nel vos cumant !" (Chanson de Roland v. 27I-273)

L'empereur répond [à l'archevêque Turpin] avec colère: "Allez vous asseoir sur ce tapis blanc! Ne prononcez pas une parole de plus si je ne vous en donne pas l'ordre!" 
Dans cet extrait amplement commenté de la Chanson de Roland, l'empereur Charlemagne s'adresse à l'archevêque Turpin qui, contre son souhait, s'est porté volontaire pour aller en ambassadeur auprès de l'ennemi. Contrairement à ce qu'on a longtemps pensé, cel palie blanc n'indique pas que le tapis est éloigné de l'empereur. L'expression marque plutôt la colère de Charlemagne envers son allocutaire, l'archevêque, à qui il donne dans le même temps l'ordre de se taire. En rejetant le référent (le tapis) hors de sa sphère, c'est donc en réalité son allocutaire que l'empereur rabroue et que, d'une certaine façon, il rejette hors de son espace personnel. L'exemple suivant nous parait pouvoir illustrer le même type de situation, même si l'opposition au destinataire est sans doute davantage sujette à interprétation:

(6) Las! de quiel hore fui je nez, quant en tel vilté me tenez que cil ribaut mastin puant, qui flatant vos vont et chuant, sunt si seigneur de vos et mestre, don seus deüsse sires estre (Roman de la Rose, v. 9195-9200)

Hélas! maudite fut l'heure où je naquis puisque vous m'humiliez ainsi, quand ces chiens puants de débauchés qui s'emploient à vous caresser et flatter sont de vous maîtres et seigneurs, alors que je devrais seul vous régenter (Traduction d'A. Lanly, Paris: Champion, I975: 38 )

Jean de Meun rapporte ici un dialogue fictif entre un mari trompé et son épouse adultère, et l'ensemble du passage vise plus largement à illustrer l'inconstance et la perfidie féminine. L'expression injurieuse cil ribaut mastin puant permet au mari de marquer tout son mépris envers les amants de sa femme en les rejetant loin de lui, mais elle peut également rabaisser celle à qui les paroles s'adressent. On sait par ailleurs la vigueur des attaques de Jean de Meun à l'encontre des femmes et la très célèbre querelle à laquelle ses propos ont donné lieu par la suite.

Dans plusieurs des énoncés examinés jusqu'ici, la série CIL a permis au locuteur de marquer son rejet de l'allocutaire, quand la série CIST lui a permis au contraire de marquer un lien personnel fort avec le référent. Dans d'autres encore (les exemples (I) et (2)), CIST indique que le référent appartient au locuteur, CIL qu'il appartient au destinataire. Nous pensons donc que si en ancien français le locuteur est toujours le repère par rapport auquel se définit le référent du SN démonstratif, le choix des séries CIL et CIST est souvent conditionné par la situation interpersonnelle des interactants dans le hic et nunc de l'énonciation. Plus précisément, le jeu sur les deux paradigmes CIST et CIL peut permettre au locuteur de dissocier explicitement ce qui relève de sa sphère personnelle (avec CIST) de ce qui relève de celle de son allocutaire (avec CIL).

Mais les contextes pragmatiques dans lesquels les sphères du locuteur et de l'allocutaire s'opposent sont relativement rares et marqués. En réalité, dans bien des cas les sphères des deux personnes de l'interlocution semblent plutôt coïncider. En particulier, les partenaires de l'interlocution partagent en général le fait de se 
trouver dans la même situation de communication. Or on constate que pour référer au lieu et au temps de l'énonciation c'est toujours la série CIST qui est utilisée:

(7) «De cest chastel ou nos sommes [...]" (Queste del saint Graal, p. 232)

(8) A la fin de cest siecle e al deerain jur, Il jugera le munt (Vie de saint Thomas Becket, v. 3563-3564) $A$ la fin de notre monde et au dernier jour, Il jugera le monde

La série CIL indique quant à elle que les lieux et les moments auxquels on réfere sont internes au récit et externes au hic et nunc de l'énonciation:

(9) il cevauchent cele matinee (Tristan en prose, p. 26) ils chevauchent ce matin-là

Ces quelques exemples montrent que le paradigme de CIST peut également être utilisé lorsque le locuteur réfere à sa propre sphère ET à celle de son interlocuteur. Dans la plupart des énoncés précédents au contraire, la sphère de l'allocutaire était plutôt associée à CIL. On en conclut que CIST permet au locuteur soit d'inclure le destinataire dans sa sphère, soit de marquer d'une certaine façon la limite entre sa sphère personnelle et celle de l'autre. Quant à CIL, il englobe parfois la sphère de l'allocutaire, mais il indique parfois aussi le domaine extérieur à la sphère de l'interlocution. D'une certaine façon, il correspond lui aussi à une zone qui inclura ou exclura selon les cas le destinataire.

Il semble donc qu'en fonction du contexte le locuteur ait le choix d'associer l'une ou l'autre série des démonstratifs à son allocutaire et qu'il se positionne ainsi vis-à-vis de lui. L'allocutaire n'est pas associé à une place fixe dans le système des déictiques et à une série de formes particulière. Sa position étant par définition variable et mouvante dans la situation de l'interlocution, c'est précisément le choix de CIL ou de CIST qui permettra au locuteur de s'associer à lui dans certains cas, de s'en dissocier dans d'autres.

Nous aimerions à présent compléter les quelques remarques qui précèdent par l'examen de nouveaux contextes d'emploi dans lesquels le référent du SN démonstratif est de nature discursive.

\section{SPHÈRE PERSONNELLE DU LOCUTEUR ET PRISE EN CHARGE DU DISCOURS PAR LE LOCUTEUR}

Nous commencerons par reprendre à Marchello-Nizia (2006a) l'analyse de quelques énoncés dans lesquels le SN entretient une relation anaphorique avec un segment de discours adjacent ou proche. Dans les exemples du type de (IO), le référent du SN démonstratif a la particularité qu'il est de nature propositionnelle:

(IO) Nos cestes pugnes non avem,

Contra nos eps pugnar devem (Passion de Clermont, v. 50I-502)

Nous, ces combats, nous n'avons pas à les mener, c'est contre nous-mêmes que nous devons nous battre 
Dans ce type d'énoncé, le SN démonstratif permet au locuteur de reprendre le contenu de la totalité ou d'une partie de son discours précédent grâce à une anaphore résomptive. Dans le cas de l'extrait de la Passion de Clermont (vers l'an Mil) donné en (IO), l'auteur résume tout le contenu du récit qu'il vient de faire de la passion du Christ à l'aide du SN cestes pugnes. Le SN cestes pugnes ne réfêre donc pas aux combats du locuteur, mais à ceux du Christ. Le choix de la série CIST montre cependant que le locuteur s'implique personnellement dans son discours au moment même où il exhorte directement son public à mener ses propres luttes. Le démonstratif de la sphère personnelle (CIST) indique que le locuteur prend en charge et assume le contenu de son propre discours quand il quitte le domaine du récit pour interpeler son auditoire.

La situation inverse est exemplifiée en (I I):

(I I) La domnizelle celle kose non contredist (Sainte Eulalie, v. 23)

La jeune fille ne s'opposa pas à cette chose.

Dans cet énoncé également tiré d'un des plus anciens textes écrits en français (vers 88I), l'expression celle kose ne fait que reprendre de façon synthétique le contenu discursif donné précédemment, c'est-à-dire l'ordre donné par le roi païen de couper la tête de la jeune sainte. Cette fois-ci, le contenu discursif auquel réfere l'anaphore résomptive celle kose n'est pas pris en charge de façon explicite par le locuteur. C'est donc la série CIL qui est utilisée.

Une autre situation, comparable, mais légèrement différente et très fréquente dans les textes médiévaux, est celle dans laquelle le SN démonstratif fait suite à un segment discursif au discours direct. Les SN démonstratifs du type aprés ceste/cele parole ou a cest/cel mot permettent de subsumer de manière synthétique le contenu du discours direct qui précède et par là-même de clore la séquence (Guillot, 2004). Ils indiquent clairement qu'on quitte le domaine de l'oral représenté pour entrer à nouveau dans la narration. Or, Marchello-Nizia (2006a et b) montre que dans la Chanson de Roland CIST est utilisé lorsque le locuteur des paroles est également le sujet syntaxique de l'énoncé qui suit:

(I2) «Quant tu es mor, dulur est que jo vif ».

A icest mot se pasmet li marchis (Chanson de Roland, v. 2030-203 I)

"Dès lors que tu es mort, c'est un malheur que je vive ". Sur ces paroles/en prononçant ces paroles le marquis tombe évanoui.

Dans le cas opposé où les paroles déjà citées n'ont pas pour auteur le référent qui correspond au sujet syntaxique de l'énoncé, c'est une forme de CIL qui est choisie:

(I3) "Sempres murrai, mais cher me sui vendut».

A icel mot l'at Rollant entendut (Chanson de Roland, v. 2053-2054)

"Je vais mourir bientôt, mais je me suis chèrement vendu!". A ces mots Roland l'a entendu. [Roland n'est pas l'auteur des paroles rapportées]

Là encore, on peut considérer que l'alternance CIST / CIL permet de mettre en relation un contenu discursif avec une source énonciative, qui s'avère être le 
locuteur dans le cas de CIST (ou plus exactement le sujet de conscience en position thématique au sein de l'énoncé), et une autre source énonciative dans le cas de CIL. D'une certaine façon, ce constat rejoint la perspective développée par P. MasséArkan dans un récent article (Massé-Arkan, 20 I I), où elle montre que la série CIST est préférentiellement utilisée dans le discours rapporté et dans la délimitation par l'auteur des plans discursifs du texte. Le paradigme de CIST permettrait ainsi de faire « coïncider normalement le centre déictique et le point de vue depuis lequel est envisagée la désignation, alors que l'emploi de CIL indique la mise en place d'un centre déictique fictif, qui va constituer une perspective narrative " (MasséArkan, 20II: 459-460). Pour notre part, nous pensons que pour bien comprendre et analyser le fonctionnement des deux séries, il est plutôt nécessaire de prendre en compte non seulement le locuteur mais aussi l'allocutaire et leur position respective dans la situation de l'énonciation. ${ }^{3}$

On sait que ce système d'alternance entre CIST et CIL après le discours direct ne fonctionne plus vraiment à l'identique après la Chanson de Roland (vers I Ioo), et, pour C. Marchello-Nizia, c'est l'un des contextes dans lesquels l'opposition sémantique entre CIST et CIL commence à se brouiller et à se perdre. On voit par ailleurs que, dans les exemples (IO) à (I 3), ce qui semble primer c'est la façon dont le locuteur se positionne par rapport à son discours plutôt que par rapport à son allocutaire. On remarque toutefois qu'en (Io) l'engagement personnel du locuteur s'accompagne d'une adresse directe à l'auditoire et d'une exhortation à agir, qui aboutit à inclure le locuteur et son destinataire dans une même collectivité (contra nos eps, " contre nous-mêmes").

Si ces exemples apportent un éclairage différent sur les stratégies communicatives du locuteur, ils présentent également l'intérêt de nous permettre d'élargir et de préciser la définition de la sphère personnelle. Il semble en effet qu'avec le démonstratif le locuteur puisse moins caractériser le lien qu'il entretient avec le référent en tant qu'objet du monde qu'en tant qu'objet de son propre discours. Nous reviendrons plus loin (section 4) sur la relation qu'on peut établir entre cet aspect de la sphère personnelle et la situation de l'interlocution. Dans l'immédiat, nous allons tenter de montrer qu'une des manières qu'a le locuteur d'associer le référent à sa personne et à sa sphère est de prendre en charge personnellement la façon dont il le nomme et le présente dans son discours.

\section{SN DÉmonstratifs prédicatifs et (NON) PRISE EN CHARGE PAR LE LOCUTEUR}

Nous nous limiterons dans cette section à l'étude de SN qui ont la particularité d'apporter des informations inédites sur le référent. Comme on l'a constaté à de nombreuses reprises (Kleiber, I984, I986a et b ; Corblin, I987), CE N permet

${ }^{3}$ La théorie de P. Masssé-Arkan peut plus difficilement rendre compte des énoncés analysés par C. Marchello-Nizia à partir de la notion de sphère personnelle du locuteur (le fait que cel cheval réfere par exemple au cheval de l'allocutaire par opposition à celui du locuteur). 


\section{Céline Guillot}

de classifier le référent comme étant un N. Dans les cas où cette classification apporte des informations qui ne sont pas déjà données dans le contexte discursif ou situationnel, on peut assimiler la (re)catégorisation opérée via le SN à une forme de prédication (Schnedecker, 2006). Nous parlerons dans ce cas de SN démonstratifs prédicatifs. Dans l'énoncé (I4) par exemple, cist merveillus champiuns présente Jesbi de Nob sous un jour nouveau:

(I4) E cume David fud alches alasséd, Jésbi de Nób, ki fud del lignage Araphá é out ceínt un brant núef é flambánt, é li fers de sa lance pesad treis cenz unces, cist merveillus champiuns volt ferir le rei David. (Quatre livres des rois, p. IOO)

Et comme David était un peu fatigué, Jesbi de Nob, qui était du lignage de Arapha et avait ceint une épée flambant neuve et avait une lance dont le fer pesait trois cents onces, ce champion merveilleux voulut frapper le roi David.

Pour illustrer notre hypothèse sur le SN démonstratif prédicatif, nous avons choisi de dépouiller deux textes du $3_{3}{ }^{\text {ème }}$ siècle, les Miracles de Notre Dame de Gautier de Coinci (vers I2 I8-I227) et le Roman de la Rose de Jean de Meun (entre I269 et I278). La quasi totalité des exemples cités dans les sections 3 et 4 sont extraits de ces deux oeuvres.

L'hypothèse que nous avons proposée pour la période médiévale (Guillot, 20 Iob) est que, dans les SN démonstratifs prédicatifs, le choix de la série CIST ou CIL dépend de la source énonciative présentée comme étant à l'origine de la prédication. Lorsque le locuteur choisit d'utiliser l'une des formes de CIST, il indique à son allocutaire qu'il assume personnellement le contenu informatif véhiculé par le SN. Quand il choisit au contraire l'une des formes de CIL, il présente la prédication opérée via le SN comme ne venant pas de lui. En d'autres termes, la série CIST comporte le trait «+ prise en charge du contenu informatif du SN par le locuteur ", la série CIL le trait opposé «- prise en charge du contenu informatif du SN par le locuteur ". Notre approche suppose donc que ce n'est plus le référent en tant que tel qui est associé au locuteur (à l'intérieur de la sphère personnelle) ou qui en est dissocié (à l'extérieur de la sphère), mais la façon de le dénommer, le locuteur ayant la possibilité d'assumer ou non la dénomination utilisée en discours.

Nous pensons que ce trait " $+/$ - prise en charge par le locuteur " est particulièrement saillant dans les SN démonstratifs prédicatifs et qu'il permet de rendre compte de la répartition particulière des séries CIST et CIL. Il permet, par exemple, de comprendre pourquoi c'est la série CIST qui est utilisée dans l'exemple suivant :

(I5) Juvenaus meïsmes escrie

a Posthumus qui se marie:

"Posthumus, veuz tu fame prandre?

Ne peuz tu pas trover a vandre

ou harz ou cordes ou chevestres [...]?

Quel forsenerie te maine

a cest torment, a ceste paine ?" (Rose v. 8705-87I4) 
Juvénal crie lui aussi à Postumus qui se marie:" Postumus, tu veux prendre femme? Ne peux-tu pas plutôt trouver des cordes, cordeaux ou licous qui soient à vendre [...] ? Quelle fureur te mène à ce tourment, à cette peine ? " (Traduction A. Lanly, t. 2, vol. 2, Paris: Champion, I975, p. 20)

Dans ces propos rapportés de Juvénal, le mariage est présenté comme étant un tourment et une peine. On peut considérer que le choix de la série CIST dans les SN a cest torment et a ceste paine indique clairement qu'il s'agit là d'un jugement porté par l'auteur antique. Mais cette thèse, à laquelle l'autorité de Juvénal apporte certainement un crédit tout particulier, est également celle du mari jaloux, qui est l'auteur du discours citant dans lequel s'insère ce discours rapporté. Enfin, il est bien possible que cette opinion soit également partagée par Jean de Meun et qu'il se serve de cette expression pour faire entendre son point de vue personnel sur le mariage.

Dans l'énoncé (I5), la figure allégorique d'Amour présente les deux auteurs du Roman de la Rose, Guillaume de Lorris et Jean de Meun son continuateur. Guillaume de Lorris apparait comme le premier serviteur d'Amour auquel Jean de Meun viendra en aide par la suite:

(I5) Vez ci Guillaume de Lorriz, Cui Jalousie, sa contraire, Fet tant d'angoisse et de deuls traire Qu'il est en perill de morir [...], Si vos cri merci, jointes paumes, Que cist las doulereus Guillaumes,

Qui si bien s'est vers moi portez, Soit secouruz et confortez.» (Rose, v. I0496 - I0630)

Voici Guillaume de Lorris à qui Jalousie, son ennemie, fait endurer tant de souffrance et de chagrin qu'il est en danger de mourir [...], aussi je vous supplie, les mains jointes, que ce malheureux, l'infortuné Guillaume qui envers moi s'est bien comporté, soit secouru et réconforté.

La prédication opérée par cist las doulereus Guillaumes n'est peut-être pas entièrement nouvelle ici, dans la mesure où elle reprend une partie des informations données dans le passage qui précède. Mais la distance qui sépare le SN de reprise du segment discursif dans lequel le référent a été introduit au départ peut laisser penser qu'il est nécessaire de réactiver ces connaissances. Par ailleurs le choix du démonstratif et des expansions du nom peut sembler motivé par la volonté du locuteur de faire preuve d'une certaine empathie personnelle avec le référent.

L'exemple (I6) montre que le locuteur peut exploiter la valeur du démonstratif pour jouer sur le contenu sémantique et axiologique du SN. Dans ce nouvel extrait du Roman de la Rose, qui rapporte les paroles prononcées par Danger lorsqu'il barre 


\section{Céline Guillot}

le chemin de l'amant s'approchant de la rose, l'expression cest biau servise semble être à la fois revendiquée par le locuteur et ironique : ${ }^{4}$

(I6) «Fuiez, vassaus, fuiez, fuiez, fuiez, dist il, trop m'annuiez.

Deable vos ont ramené, li malaaist, li forsené, qui a cest biau servise partent, ou tout preingnent ainz qu'il s'en partent! (Rose, v. I4796-I4802) "Fuyez, jeune-homme, fuyez, fuyez, fuyez, dit-il, trop vous m'importunez. Ce sont les diables qui vous ont ramené, ces maudits, ces enragés qui participent à ce beau service: qu'ils emportent tout [et vous avec!] avant de s'en aller!" (Traduction Lanly, p. 9I)

Le fait que l'assertion véhiculée par le SN soit prise en charge par le locuteur permet en même temps de mettre en évidence son caractère problématique et la valeur antiphrastique prise par le $\mathrm{SN}$ en contexte.

Comme nous l'avons vu précédemment, la série CIL est au contraire utilisée lorsque le locuteur n'assume pas personnellement le contenu notionnel du SN. Nous avons essayé de montrer ailleurs (Guillot, 20Iob) qu'alors le contenu de ce SN n'est généralement pas nouveau. Il a été donné déjà dans l'entourage discursif et le SN n'est pas pleinement prédicatif. ${ }^{5}$

Dans les contextes où le SN semble toutefois informatif, nous proposons de considérer que CIL $\mathrm{N}$ présente la prédication comme reposant sur des connaissances partagées par le locuteur et son destinataire. Autrement dit, la catégorisation du référent est donnée par le locuteur comme faisant l'objet d'un sorte de consensus et non pas comme étant la sienne propre. Ainsi s'explique selon nous que ce soit la forme (i)cil qui est utilisée dans cet extrait du Jugement de Renart :

(I7) «Et je sai bien, que que nus die, que cil qui tot le mont conchie, Renart, icil mavés lechierres, cil rous puanz, cil orz trichierres,

eüst ma fame si baillie [...]

Certes, ce fu mout grant damajes c'onques Renart, cil fel, cil rous, vos bati onques le velous. " (Renart, branche I, v. 89-IO2)

Et je sais bien, quoi que l'on dise, que celui qui conchie tout le monde, ce sale rouquin, ce pendard infâme, s'il avait traité ma femme de la sorte... Quel malheur que Renart, ce bandit, ce rouquin, vous ait ainsi brossé le poil!" (Traduction H. Rey-Flaud et A. Eskénazi, Paris: Champion, I982, p. 23)

${ }^{4}$ Lanly indique en note: «ce beau service, au vers I480I, est évidemment antiphrastique " (p. I90).

5 C'est notamment le cas dans la reprise fidèle qui nous semble être très majoritaire avec CIL. Ce point reste à préciser grâce à une étude quantitative en cours. 
Le fait de présenter les qualificatifs associés à Renart comme reflétant l'opinion commune donne d'autant plus de poids à l'accusation qui est portée contre lui par le locuteur. De la même façon, dans l'exemple (I8), Gautier de Coinci lance ses invectives contre les Juifs responsables de la mort d'un jeune-homme. En utilisant les expressions cil granz murdres, cil granz pechiez, il présente la mort du jeune garçon comme le résultat du meurtre et du forfait de ceux qu'il prend pour cible. Le fait de présenter ces deux assertions comme admises par tous pourrait être une manière de prendre le lecteur/auditeur à partie :

(I8) Mout sont dolant clerc et provoire

Dou biau clerçon qui est peris [...].

\section{Cil granz murdres, cil granz pechiez,}

Longues ne puet estre celés (Gautier de Coinci, II Mir I3, t. 4, v. 318, p. 54)

Les clercs et les prêtres sont très affligés de la mort du beau jeune homme [...]. Ce grand meurtre, ce grand péché ne put être caché longtemps.

Le caractère polémique des extraits (I7) et (I8) nous ramène ainsi à la question posée plus haut: en quoi l'utilisation des démonstratifs nous renseigne-t-elle sur la position relative des deux partenaires de l'interlocution ? En quoi le choix de l'une ou l'autre des deux séries médiévales est-il conditionné par la façon dont ils se situent l'un par rapport à l'autre ? En examinant ces différents points, notre objectif sera également d'étayer notre analyse des énoncés examinés dans cette section (cette analyse peut, à certains égards, sembler ad hoc), en la confrontant à d'autres exemples, à première vue assez différents et dont l'interprétation parait moins discutable.

\section{SN DÉmONSTRATIFS PRÉdiCATIFS ET STRATÉGIES COMMUNICATIVES}

Pour mettre en relation l'usage des démonstratifs et la position des partenaires de l'interlocution, nous nous intéresserons aux types et genres discursifs des textes de notre corpus. Les deux textes que nous avons dépouillés, les Miracles de Gautier de Coinci et le Roman de la Rose de Jean de Meun, ont une particularité commune: en bien des endroits, leur visée est argumentative et l'auteur cherche à convaincre son lecteur (ou son auditoire) du bien fondé de son point de vue. Il ne s'agit donc pas d'oeuvres narratives au sens où le sont la plupart des romans par exemple. ${ }^{6}$ Gautier de Coinci et Jean de Meun passent en effet beaucoup de temps à dénoncer ce qu'ils considèrent comme des abus chez leurs contemporains et leurs ouvrages font une large place à la satire.

${ }^{6}$ Nous ne prétendons pas ici donner une caractérisation précise et complète de chacune de ces oeuvres. On considère généralement le Roman de la Rose comme une encyclopédie laique (voir notamment Jauss, I986: 44), et les recueils de miracles constituent un genre discursif à part entière dont le but est avant tout l'édification religieuse et morale des fidèles. Ce sont plutôt les passages dans lesquels on rencontre les formes qui nous intéressent qui nous paraissent relever du type argumentatif. 
Nous proposons donc de mettre en relation l'usage de CIL et de CIST dans ces deux oeuvres avec la visée argumentative des segments discursifs dans lesquels ils se trouvent. Nous avons vu dans la section précédente un ensemble d'occurrences de CIST $\mathrm{N}$ qui marquent l'implication de l'auteur dans son propre discours. Nous proposons de considérer par exemple qu'en assertant quel forsenerie te maine a cest torment, a ceste paine Jean de Meun peut à la fois présenter le jugement de Juvénal sur le mariage mais aussi asserter son point de vue personnel face à son lecteur. Nous nous centrerons ici au contraire sur des $\mathrm{SN}$ qui ne sont pas pris en charge par l'auteur, bien qu'ils comportent eux aussi un contenu axiologique. Il s'agit de SN génériques au pluriel, qui réferent à un groupe d'individus et le disqualifient de manière très explicite :

(I9) Pappelardie est une trueve

Et une gille toute nueve

Que trovee ont cil guilleeur

\section{Et cil soutil bareteeur}

Por demener tres soutilment

Leur guille et leur concïement. (Gautier de Coinci, t. 2, I Mir. I I, v. $1493-1498$, p. 62)

L'hypocrisie (fausse dévotion) est une trouvaille et une tromperie toute neuve qu'ont trouvée ces trompeurs et ces fourbes subtils pour exercer très subtilement leur tromperie et leur imposture

(20) Je voi, se Diex me doinst honeur,

Que cil las, cil fratre meneur

Qui par ces voies vont tranblant,

Font bele chiere et bel samblant

Et belement as gens parolent,

Mais cil begin d'ire m'afolent.

\section{Cil pappelart, cil ypocrite}

Une chiere font si afflite

Que par samblant se font plus juste (Gautier de Coinci, t. 2, I Mir. I I, v. $1427-1435$, p. 60)

Je vois, aussi vrai que j'invoque l'estime de Dieu, que ces misérables, ces frères mineurs qui vont par les chemins en tremblant, ont belle mine et belle apparence et parlent aux gens doucement, mais ces béguins me rendent fou de colère. Ces faux dévôts, ces hypocrites, font une mine si affligée qu'en apparence ils se font plus justes

(2 I) [...] un jor avint

Qu'en la rue des gïus vint

La chaitive, paumes batant [...] :

«Fiex doz, murtri et acoré

T'ont cil gïu, cil puant chien [...]» (Gautier de Coinci, t. 4, II Mir. I3, v. $335-347$, p. 56)

Un jour arriva où vint dans la rue des Juifs la malheureuse, battant les mains:

"Cher fils, ces Juifs, ces chiens puants, t'ont meurtri et abattu " 
(22) Qui tout le mont aroit conquis,

Se fust perdus, qu'aroit conquis?

Cil userier, cil robeür,

Cil avocat, cil plaideür,

Qui ja ne querroient finer

De tolir et d'acrapiner,

Que feront il, biaus sire Diex? (Gautier de Coinci, t. 2, I Mir. I9, v. 5I9525, p. I77-I78)

Celui qui aurait conquis le monde entier, s'il était perdu, qu'aurait-il conquis ? Ces usuriers, ces voleurs, ces avocats, ces plaideurs, qui ne croiraient jamais finir de prendre et d'arracher, que feront-ils, cher seigneur Dieu?

Assez fréquentes dans ces deux textes, les expressions de ce type nous paraissent avoir été choisies dans le souci de susciter l'adhésion du destinataire: en présentant le référent sous un jour inédit mais en n'assumant pas la responsabilité des jugements de valeur qu'il porte sur lui, l'auteur donne à penser que ces jugements sont connus et admis de tous, et tout particulièrement de celui auquel il s'adresse.

Dans d'autres cas, le SN démonstratif n'est pas directement prédicatif. Mais nous nous demandons si le fait d'utiliser la forme cil n'est pas une fois encore une manière de présenter la prédication véhiculée par l'énoncé comme une vérité connue de tous:

(23) Biaus sire Diex, glorieus pere,

Con font hui de ton patremoigne

\section{Cil riche clerc, cil haut chanoigne}

Grans degras et grans godemines. (Gautier de Coinci, t. 2, I Mir. I I, v. IOO8-IOI I, p. 44)

Cher seigneur Dieu, père glorieux, comme ces riches clercs, ces hauts chanoines, font aujourd'hui de grandes réjouissances et de grandes ripailles de ton patrimoine

On note que, dans tous ces extraits, l'auteur dénonce les pratiques d'une catégorie ou d'une classe sociale prise dans son ensemble (le référent est générique). Il ne s'agit pas pour le locuteur de se positionner personnellement face au destinataire, mais plutôt d'utiliser une stratégie communicative adaptée à la visée argumentative de son discours.

On pourrait être tenté de considérer que, dans les exemples (I9) à (23), l'usage de la série en $-\mathrm{L}$ est conditionné par le rejet explicite du référent visé. CIL marquerait dans ce cas l'exclusion du référent hors de la sphère du locuteur, comme le faisaient les SN cel palie blanc ou celle teste dans les contextes cités dans la section I. Mais cette hypothèse occulterait le rapprochement possible avec d'autres types de SN en CIL, qui sont pourtant dépourvus de toute valeur dépréciative et qu'à la suite de Kleiber (I987 et I990) nous considérons comme des occurrences du démonstratif de notoriété. 


\section{DÉmonstratif DE NOTORIÉté ET STRATÉGIES COMMUNICATIVES}

Pour la période médiévale, le démonstratif de notoriété est utilisé dans deux contextes principaux. Dans le premier cas, CIL N est déterminé par une proposition relative (exemples 24 et 25 ). G. Kleiber a bien montré que cette construction, impossible avec la série CIST, présente le référent et le contenu de la relative comme étant déjà connus de l'allocutaire:

(24) « [...] cil Dex qui ne mentit Voz puist garir [...] (Ami et Amile, v. I439-I440)

"Puisse Dieu, qui n'a jamais menti, vous protéger "

(25) «[...] Par cel apostre qu'on quiert en pré Neiron [...]» (Cour. de Louis, v. 1797)

"Au nom de l'apôtre qu'on vénère dans les jardins de Néron " ${ }^{7}$

Ces expressions figées apparaissent en assez grand nombre dans la littérature médiévale et on ne saurait imaginer qu'elles puissent marquer un quelconque rejet du référent

Ce premier type se distingue de celui auquel on attribue plus communément le nom de démonstratif de notoriété et qu'on appelle parfois aussi démonstratif épique parce que c'est surtout dans le genre de l'épopée qu'il se rencontre :

(26) Ce fu en mai el novel tens d'esté ;

Florissent bois et verdissent cil pré,

Ces douces eves retraient en canel,

Cil oisel chantent doucement et soëf. (Prise d'Orange, v. 39-42)

C'était en mai, au retour de la belle saison;

les bois fleurissent, les prés verdissent,

les douces eaux se retirent dans leur lit,

les oiseaux chantent doucement et harmonieusement (traduction C. Lachet et J.-P. Tusseau, Paris : Klincksieck, 2002)

On a bien montré déjà (Guiraud, I967 ; Wilmet, I979 ; Marchello-Nizia, I979: I 56 ; Kleiber, I990 ; Ménard, I994: 32) que ce démonstratif est employé dans des séquences textuelles stéréotypées et qu'il sert à mentionner un référent attendu dans un discours conventionnel. Dans le cas de l'exemple (26), on considère que le chant des oiseaux fait partie de la description habituelle du printemps. On présente donc ces éléments comme étant d'une certaine façon déjà connus du public.

Ce n'est donc pas l'identité du référent, qui est d'ailleurs toujours générique, qui est connue du destinataire du message. Mais c'est en tant qu'élément typique d'une séquence textuelle (une description du printemps par exemple), que le référent est donné comme faisant partie des connaissances partagées du locuteur et de celui

7 Dans cette formule très répandue dans les chansons de geste, l'identification du référent repose sur la connaissance largement répandue au Moyen Age que l'apôtre que l'on vénère dans les jardins de Néron est Pierre. 
à qui il s'adresse. Ainsi s'explique qu'on puisse utiliser un SN démonstratif pour référer à un élément qui est totalement nouveau dans le discours et absent du contexte situationnel. C'est le caractère convenu du discours, qui convoque des topoi largement répandus dans les textes de cette période, qui rend possible l'usage du démonstratif, mais on peut considérer également que le démonstratif participe de ce caractère conventionnel en donnant au discours un aspect de déjà vu, déjà connu du locuteur et de son destinataire.

On voit que le démonstratif de notoriété (exemplifié en (24) - (26)) sert toujours à caractériser le référent comme étant connu à la fois du locuteur et de son destinataire, soit parce que sa notoriété est universelle - c'est le cas de Dieu et de l'apôtre Pierre - soit parce qu'il est considéré par le locuteur comme faisant partie de l'horizon d'attente de son public dans la situation de l'énonciation. Or dans tous ces cas, la série CIL est la seule à pouvoir être employée.

Nous proposons de rapprocher ces contextes d'occurrence de CIL de ceux que nous venons de voir dans la section précédente. Chaque fois, c'est le fait que l'information est partagée, commune aux partenaires de l'interlocution, qui est mis en avant avec CIL, et c'est ce qui crée une relation de proximité entre le locuteur et le destinataire ${ }^{8}$. Ainsi s'explique l'effet de connivence (Himmelmann, I996 ; GaryPrieur, 1998 et 2003 ; Cornish, 200I) provoqué par tous ces énoncés. Dans le cas des chansons de geste par exemple, on sait qu'elles faisaient l'objet de performances devant un public collectif de cour. Elles célèbrent les prouesses chevalresques de la noblesse à qui elles s'adressent. L'usage parfois massif du démonstratif de notoriété dans ce genre discursif pourrait être lié à cette recherche d'une complicité avec le public dans la situation de la performance (Guillot, 20IOa), le démonstratif de notoriété permettant d'associer collectivement les membres de l'auditoire à un discours dans lequel ce public se reconnait et qu'il est invité à partager.

\section{CONCLUSION PROVISOIRE pOUR LA période Médiévale}

L'analyse d'un ensemble de textes diversifiés de la période médiévale, nous paraît avoir montré que l'usage des démonstratifs peut être conditionné par des stratégies communicatives et interactionnelles et par un jeu de places qui se construit en discours. Nous espérons avoir montré que le choix de CIST et CIL est l'un des moyens utilisés par le locuteur pour se positionner par rapport à son interlocuteur

${ }^{8}$ Le démonstratif de notoriété est également utilisé en français moderne dans des constructions à relative, du type Ces $N$ qui. Les études de Gary-Prieur (I998, 200I, 2003) et Kleiber (2005, 2004a et b) montrent que chaque fois le contenu de la relative, qui permet l'identification et la caractérisation du référent, est bien présenté comme étant connu du locuteur (notoire) ou familier (récupérable grâce à son expérience personnelle). Nous voudrions insister pour notre part, à la suite de M.-N. Gary-Prieur (200I: 32) sur le caractère partagé des connaissances impliquées, qu'elles soient de nature encyclopédique ou qu'elles relèvent de l'expérience personnelle: «le locuteur fait comme si le destinataire partageait avec lui la connaissance de la classe discursive dont il pose l'existence dans le GN démonstratif » (200I: 34). 


\section{Céline Guillot}

ou par rapport à son message, dans le but d'obtenir une certaine réaction de son interlocuteur.

Selon les situations de communication, les relations interpersonnelles des interlocuteurs et la visée communicative du discours, ces places peuvent évidemment varier. L'allocutaire est dans une position particulièrement instable, puisqu'il se situe tantôt du côté du locuteur tantôt à l'opposé, et c'est précisément sa position qui peut être établie et négociée en discours. On ne saurait donc défendre l'idée qu'il est associé à un seul paradigme de formes dans le système des démonstratifs médiévaux. Si l'on prend l'exemple de la série CIL, on voit qu'elle permet au locuteur tantôt de rejeter son allocutaire, tantôt de se l'associer: dans les situations d'agression ou de tension par exemple, CIL permet au locuteur de marquer son hostilité envers le destinataire (cf. l'exemple de Charlemagne rabrouant l'archevêque Turpin), dans d'autres situations au contraire, lorsqu'il s'agit par exemple de convaincre ou de récréer le public, l'emploi de CIL peut être pour le locuteur une manière de le faire adhérer à ses propres thèses ou de l'impliquer dans son discours.

Si ce jeu de places est uniquement un effet de l'usage des démonstratifs, il ne nous semble pas impossible de soutenir que c'est cet effet pragmatique et interactionnel qui conditionne souvent le choix de l'une ou l'autre série en français médiéval. Et pour aller plus loin, nous voudrions à présent établir un parallèle avec certains emplois de démonstratifs de l'anglais et du français modernes.

\section{DÉmONSTRATifS ÉmotionNel EN FRANÇAis MOderne ET STRATÉGIES COMMUNICATIVES}

Nous nous intéresserons dans cette section à ce qu'on a parfois appelé le démonstratif émotionnel (Lakoff, I974), ou démonstratif de sympathie (Chen, I990: I49-I5I). Caractéristique des interactions orales, ce démonstratif est généralement mis en relation avec l'engagement émotionnel du locuteur (" emotional involvement") dans son propre discours. C'est aux effets que cette implication personnelle peut avoir sur l'interlocuteur que l'on s'intéressera surtout ici.

L'un des multiples exemples étudiés par Lakoff concerne certaines occurrences de that qui créent une forme de solidarité entre le locuteur et le destinataire. Dans les entretiens thérapeutiques par exemple, les énoncés du type de (27):

$$
\begin{aligned}
& \text { "How's that throat?" } \\
& \text { "Comment va cette gorge?" }
\end{aligned}
$$

permettent au médecin d'indiquer à son patient qu'il se met de son côté et lui manifeste compréhension et sympathie.

En revenant sur cet exemple, Chen (I990) insiste sur le fait que ce type d'énoncé n'est possible que si le médecin a déjà connaissance des problèmes qu'a le patient avec sa gorge. Il semble donc que dans cet usage qui semble spécifique 
au pronom/déterminant that de l'anglais, ${ }^{9}$ le démonstratif est également associé à l'expression d'une connaissance commune au locuteur et à l'allocutaire. Et le fait de choisir cette forme spécifique de déterminant a pour effet de créer une certaine complicité entre les partenaires de l'interaction. On peut se demander si là n'est pas la raison du choix du démonstratif, plutôt que du possessif ou d'une autre expression référentielle.

Des recherches menées dans un tout autre cadre et portant sur l'association du démonstratif avec le nom propre en français ont mené à des constats qui ne sont pas sans rapport avec les points abordés jusqu'ici. G. Kleiber (I99I et I994), M.-N. Gary-Prieur (I994) et K. Jonasson (1994) s'intéressent aux énoncés du type de (28)

(28) Ce salaud de Victor! Il m'a encore bu tout le whisky! (cité dans Kleiber, I994: 82)

et distinguent au moins trois traits caractéristiques de cette construction: elle comporte une marque exclamative qualifiante (positive ou négative), un adjectif ou un nom évaluatif ou affectif antéposé (pauvre, bon sacré, brave, cher, etc.) et repose sur une qualification déictique du référent. On veut dire par là que la caractérisation du référent s'appuie sur des informations accessibles dans la situation de communication. L'énonciation de (28) n'est possible que si le destinataire a les moyens, dans la situation de l'échange verbal, de récupérer les éléments qui fondent le jugement du locuteur. Là encore, le démonstratif permet au locuteur de faire appel à son allocutaire et à la connaissance qu'il a du référent, dans la situation où il se trouve, pour interpréter l'énoncé. On peut se demander si l'un des intérêts de tels exemples n'est pas qu'ils nous conduisent à nous détourner de la question de l'identification du référent, que le nom propre suffit à lui seul à rendre possible en (28), pour rechercher quelle est la valeur pragmatique et interactionnelle du démonstratif dans ce contexte..$^{10}$ Et de ce point de vue, nous nous demandons si un énoncé comme (28) n'a pas lui aussi pour fonction de susciter l'implication et

${ }^{9}$ On note que, dans le système à deux termes de l'anglais, that occupe une position comparable à celle de CIL en ancien français. R. Lakoff analyse également des emplois émotionnels de this et constate que l'effet de proximité ou de camaraderie qu'il provoque est très proche de celui qu'on associe à that: «A problem we shall find in examinig that is that, while its spatio-temporal uses are very nearly opposite those of this, its emotive uses are surprisingly close" (Lakoff, I974: 349). Cette remarque invite naturellement à continuer les recherches sur ce qui distingue this et that dans les interactions verbales. Mais elle confirme d'une certaine façon que l'usage des démonstratifs est parfois (souvent ?) conditionné par des stratégies communicatives et pragmatiques, puisqu'il semble que les deux formes aient des emplois " émotionnels".

${ }^{10}$ Il s'agit pour G. Kleiber d'une des caractéristiques des combinaisons $\mathrm{CE}+\mathrm{Np}$ de ce type. Elles constituent selon lui des expressions référentielles " hybrides » qui assurent à la fois l'identification référentielle du référent et sa qualification (voir notamment Kleiber, I994: $82)$. 
l'adhésion du destinataire. ${ }^{11}$ La question se pose également pour les énoncés tels que (29), dans lesquels le jugement porté par le locuteur reste implicite:

\section{(29) Cette Louise !}

CE $\mathrm{Np}$ apparait alors comme une 'sorte de commentaire minimum d'une situation où le référent du nom propre est l'objet d'un jugement, favorable ou défavorable, de l'énonciateur' (Jonasson, I994: 2 I0). C'est donc en fonction de la situation de communication et de ce qu'il sait du référent que le destinataire est invité à interpréter l'énoncé. Nous nous demandons à nouveau si de tels exemples ne répondent pas à la même intention du locuteur: en énonçant un jugement dont la valeur reste implicite, il donne à entendre à son interlocuteur qu'ils partagent le même point de vue sur le référent. ${ }^{12}$

Pour clore cette section, nous reviendrons sur un exemple bien connu et étroitement associé à une bande dessinée célèbre :

(30) Ils sont fous, ces Romains.

On a insisté sur le fait que cette exclamation arrive toujours en fin d'épisode et sur le caractère déictique de la généralisation opérée par l'énoncé: "s'il y a renvoi déictique possible à la classe générique des Romains par le SN démonstratif Ces Romains, c'est parce que des Romains spécifiques viennent d'accomplir une action jugée "folle » ( Kleiber, I99I: 95). Mais ce clin d'oeil au public est par ailleurs tout à fait attendu et il sert de conclusion immuable au récit des aventures gauloises. D’une certaine façon, le locuteur de ce jugement - en général Obélix - fait lui aussi appel à la connaissance qu'a le lecteur de la bande dessinée et à sa complicité. Le jugement porté sur les Romains (mais aussi les Goths, les Egyptiens, les Bretons, etc.) n'apporte en soi aucune information nouvelle, il vise uniquement à conforter le lecteur dans l'idée qu'il avait déjà et dans laquelle toute une communauté peut se retrouver.

11 G. Kleiber insiste quant à lui sur l'engagement du locuteur marqué par l'usage du démonstratif. Le démonstratif indiquerait en effet "la responsabilité du locuteur dans la qualification attribuée ", tandis que l'article défini, également possible dans ce type d'énoncé, tendrait "plutôt à présenter cette même qualification comme objective, établie, acceptée " (Kleiber, I994: 83). Il nous semble que pour le français moderne nos deux interprétations ne s'excluent pas. Il est très possible que tout en assumant la responsabilité du jugement qu'il énonce le locuteur cherche en même temps à s'associer l'allocutaire dans le contexte de l'énonciation. Une étude plus poussée sur corpus pourrait nous aider à préciser ce point.

12 Bien d'autres types d'énoncés semblent répondre à la même stratégie communicative, notamment les tours exclamatifs du type Elle a une des ces santés! étudiés notamment par M.-N. Gary-Prieur: "la structure un de ces N... ! introduit donc quelque chose de plus qu'une simple valeur intensive: elle implique une complicité entre les interlocuteurs, le locuteur faisant comme si le destinataire savait reconstruire la sous-catégorie à parcourir ". (2003: 225). 


\section{CONCLUSION}

L'étude que nous venons de présenter s'est focalisée sur quelques contextes d'occurrence du démonstratif en français. En explorant et en analysant des usages limités, mais relativement peu étudiés, on a essayé de montrer que le choix des démonstratifs en ancien français est souvent motivé par des stratégies communicatives. En considérant que le démonstratif conserve une valeur personnelle en français médiéval, centrée sur la personne du locuteur, on s'est intéressé au contexte pragmatique, aux relations interpersonnelles des interactants et à la visée communicative des énoncés dans lesquels il est employé. On a ainsi essayé de montrer que le choix de l'une ou l'autre série permet au locuteur de se positionner de manière dynamique par rapport à celui à qui il s'adresse. Ce faisant, nous avons adopté une approche pragmatique, centrée sur l'interaction verbale, dans la lignée d'autres travaux menés récemment sur les démonstratifs dans les langues du monde (Enfield, 2003). Nous avons précisé la notion de sphère personnelle du locuteur et montré qu'il est possible de saisir de manière homogène des emplois épars (démonstratifs prédicatifs, démonstratifs de notoriété, démonstratifs émotionnels) dès lors que les $\mathrm{SN}$ démonstratifs sont dotés du trait " $+/$ - prise en charge par le locuteur".

Pour le français moderne, cette approche peut conduire à considérer les démonstratifs sous un angle peut-être un peu différent de celui qui domine la recherche aujourd'hui. Bien qu'on ne puisse prétendre que le système du français moderne continue de reposer de la même façon qu'en ancien français sur la personne du locuteur, nous pensons qu'on gagnerait à mettre en relation son usage avec les stratégies communicatives des personnes du discours. En cela, nous nous inspirons des travaux de M.-N. Gary-Prieur qui a toujours très explicitement mis en relation le démonstratif et les personnes de l'interlocution. ${ }^{13}$

Adresse pour correspondance:

Céline Guillot

ICAR

Ecole Nationale Supérieure de Lyon

15 parvis René Descartes - BP 7000

69342 LYON Cedex o7

France

e-mail : celine.guillot@ens-lyon.fr

13 On mentionnera pour mémoire les citations suivantes: "Ce $\mathrm{N}=$ un $\mathrm{x}$ qui est un $\mathrm{N}$ et sur lequel je attire l'attention de $t u$. " (Gary-Prieur, I998: I 8) ; "le démonstratif a donc pour effet (quelle que soit la réalisation de cet effet, qui dépend de la particularité du discours) de faire appel au destinataire. " (Gary-Prieur, I998: I9) ; "En faisant apparaitre explicitement je et $t u$, cette définition comporte l'hypothèse que $c e$ donne l'instruction de situer le référent du GN ce N par rapport aux personnes du discours. " (Gary-Prieur, 200I: 223). 


\section{B I B L I O G R A P H IE}

Chen, R. (1990). English demonstratives: A case of semantic expansion. Language Sciences, I2 / 2 et 3: I39-I53.

Corblin, F. (1987). Indéfini, défini, démonstratif. Genève-Paris: Droz.

Cornish, F. (200I). 'Modal' that as determiner and pronoun: the primacy of the cognitive-interactive dimension. English Language and Linguistics, 5/2: 2973 I 5 .

Enfield, N. J. (2003). Demonstratives in space and interaction: data from Lao speakers and implications for semantic analysis. Language, 79 / I: 82-I I 7 .

Gary-Prieur, M.-N. et Léonard, M. (1998). Les démonstratifs dans les textes et dans la langue. Langue française, I20: 5-20.

Gary-Prieur, M.-N. (1994). Grammaire du nom propre. Paris: PUF.

Gary-Prieur, M.-N. (I998). La dimension cataphorique du démonstratif. Etude de constructions à relative. Langue française, I20: 44-50.

Gary-Prieur, M.-N. (200I). GN démonstratifs à référence générique: une généralité discursive. Journal of French Language Studies, I I / 2: 22 I-239.

Gary-Prieur, M.-N. (2003). La distinction d'un élément dans une classe discursive. Etude des GN de la forme un de ces N qui P. Dans: B. Combettes, C. Schnedecker et A. Theissen (dir.), Ordre et distinction dans la langue et dans le discours. Actes du colloque international de Metz (18, 19, 20 mars 1999). Paris: Champion, pp. 2 I7-23 I.

Guillot, C. (2004) Ceste parole et ceste aventure dans la Queste del Saint Graal, marques de structuration discursive et transitions narratives. L'Information grammaticale, IO3: $29-36$.

Guillot, C. (20 IOa) Le démonstratif de notoriété de l'ancien français: approche textuelle. Dans: B. Combettes et al. (dir.), Le changement en français. Etudes de linguistique diachronique. Bern/Berlin/Bruxelles: Peter Lang, pp. 217-233.

Guillot, C. (20IOb) Les démonstratifs de l'ancien français: un système encore personnel ?, $2^{\mathrm{e}}$ Congrès Mondial de Linguistique Française, La Nouvelle-Orléans, I2-I 5 juillet de 20IO, CDrom.

Himmelmann, N. (1996). Demonstratives in narrative discourse: a taxonomy of universal uses. Dans: B. Fox (dir.), Studies in Anaphora. Amsterdam/Philadelphia: John Benjamins, pp. 205-254.

Jonasson, K. (1994). Le nom propre. Constructions et interprétations. Louvain-la-Neuve: Duculot.

Kleiber, G. (1984). Sur la sémantique des descriptions démonstratives. Linguisticae Investigationes, VIII: $63-85$.

Kleiber, G. (1985). Sur la spécialisation grammaticale des démonstratifs en français ancien. Dans: Mélanges H. Naïs, De la plume d'oie à l'ordinateur, Verbum, pp. 99I I 3 .

Kleiber, G. (1986a). Déictiques, embrayeurs, 'token-reflexives', symboles indexicaux, etc., comment les définir ? L'information grammaticale, 30: 3-22.

Kleiber, G. (I986b). Pour une explication du paradoxe de la reprise immédiate UN Ni LE Ni / UN Ni CE Ni. Langue française, 72: 54-79.

Kleiber, G. (1987). L'opposition cist/cil en ancien français ou comment analyser les démonstratifs? Revue de linguistique romane, 51: 5-35.

Kleiber, G. (I990). Sur le démonstratif de notoriété en ancien français. Revue québécoise de linguistique, I9/I: I I-32. 
Kleiber, G. (I99I). Du nom propre non modifié au nom propre modifié: le cas de la détermination des noms propres par l'adjectif démonstratif. Langue française, 92: 82-IO3.

Kleiber, G. (1994). Nominales. Essais de sémantique référentielle. Paris: Armand Colin: 66-9I.

Kleiber, G. (2004a). Sémantique, référence et discours : le cas des démonstratifs cataphoriques spécifiques. Dans: A. Auchlin et al. (dir.), Structures et discours. Mélanges offerts à Eddy Roulet. Québec: Editions Nota bene, pp. 23 I-245.

Kleiber, G. (2004b). Anticipation, mémoire et démonstratifs cataphoriques. Dans: R. Sock et B. Vaxelaire (dir.), L'anticipation à l'horizon du présent. Sprimont: Pierre Mardaga Editeur, pp. $22 \mathrm{I}-236$.

Kleiber, G. (2005). Des démonstratifs bien énigmatiques: les démonstratifs cataphoriques génériques. Dans: C. Dobrovie-Sorin (dir.), Noms nus et généricité. Paris: Presses universitaires de Vincennes, pp. 65-95.

Lakoff, R. (1974). Remarks on this and that. Proceedings of the Chicago Linguistics Society. Chicago: $345-356$.

Marchello-Nizia, C. (2003). 'Se voz de ceste ne voz poéz oster, Je voz ferai celle teste coper.' (Ami et Amile 753): La sphère du locuteur et la déixis en Ancien français. Dans: A. Vanneste, P. De Wilde, S. Kindt et J. Vlemings (dir.), Memoire en temps advenir. Hommage à Theo Venckeleer. Louvain-Paris: Peeters, pp. 4I3-427.

Marchello-Nizia, C. (2004). La sémantique des démonstratifs en ancien français: une neutralisation en progrès? Langue française, I $4 \mathrm{I}$ : $69-84$.

Marchello-Nizia, C. (2005). Deixis and subjectivity: the semantics of demonstratives in Old French (9th-I2th century). Journal of Pragmatics, 37/I: 43-68.

Marchello-Nizia, C. (2006a). Du subjectif au spatial: l'évolution des formes et du sens des démonstratifs en français. Langue française, I 52: I I 4-I 26.

Marchello-Nizia, C. (2006b). From personal to spatial deixis: The semantic evolution of demonstratives from Latin to French. Dans: M. Hickman et S. Robert (dir.), Space in languages, linguistic systems and cognitive categories. Amsterdam/Philadelphia: Benjamins Publishing Company, pp. I03-I20.

Massé-Arkan, P. (2O I I). Les démonstratifs cil et cist en ancien français: le livre et l'espace du récit. Romania, I29: 427-460.

Schnedecker, C. (2006). SN démonstratifs 'prédicatifs': qu'est-ce qui limite leur apport informatif? Langue française, I 52: 39-55.

\section{TEXTES CITÉS}

Ami et Amile, édité par P.F. Dembowski, Paris: Champion (CFMA 97), I969.

Chanson de Roland, édité par G. Moignet, Paris: Bordas, I969.

Gautier de Coinci, Miracles, édité par V. F. Koenig, Genève: Droz (TLF 64, 95 I3 I, I76), I955-1970.

Guernes de Pont-Sainte-Maxence, Vie de saint Thomas Becket, édité par E. Walberg, Paris: Champion (CFMA 77), I936.

Jean de Meun, Roman de la Rose, édité par F. Lecoy, Paris: Champion (CFMA 92, 95, 98), 1965-I970.

Le couronnement de Louis, édité par E. Langlois, Paris: Champion (CFMA 22), I925. 
Passion de Jésus-Christ ou Passion de Clermont, édité par A. S. Avalle, Milan: Riccardo Ricciardi, 1962.

Prise d'Orange, édité par C. Régnier, Paris: Klincksieck, I972.

Quatre livres des Rois, édité par E. R. Curtius, Dresde: Max Niemeyer, I9I I.

Queste del saint Graal, édité par A. Pauphilet, Paris: Champion (CFMA 33), I923.

Roman d'Eneas, tome I, édité par J. Salverda de Grave, Paris: Champion (CFMA 44), I925.

Roman de Renart, branche I, édité par M. Roques, Paris: Champion (CFMA 78), I948.

Séquence de sainte Eulalie, édité par R. L. Wagner et O. Collet, Textes d'études, Genève: Droz (TLF 460), I995.

Tristan en prose, tome I, édité par P. Ménard, Genève: Droz (TFL 353), I987. 